\title{
Implementação de fantoma geométrico de mão e antebraço no código de Monte Carlo GEANT4
}

\author{
P. R. Pessanha a; P. P. de Queiroz-Filho ${ }^{\text {a }}$ D. Souza-Santos ${ }^{\text {a }}$ \\ ${ }^{a}$ Instituto de Radioproteção e Dosimetria - IRD/CNEN, CEP: 22783-127, Rio de Janeiro-RJ, Brasil \\ pessanha.paular@gmail.com
}

\begin{abstract}
RESUMO
Anualmente, diversos radiofármacos são utilizados em mais de 50 procedimentos clínicos diferentes em todo o Brasil. Sendo assim, profissionais inseridos nas diversas práticas de Medicina Nuclear que envolvem atividades ligadas a radio-emissores de alta energia representam um grupo de maior exposição à radiação e justificam esforços permanentes na otimização de riscos radiológicos. A utilização de técnicas avançadas de monitoração representa um importante subsídio para a avaliação da exposição ocupacional em instalações e a consequente redução das doses individuais. Neste trabalho foi feita a implementação de um fantoma de mão e antebraço em código Geant4, para a avaliação da exposição ocupacional de extremidades de trabalhadores que realizam práticas de Medicina Nuclear. O modelo de simulação oferecido pelo Geant4 inclui um conjunto completo de recursos, com a reconstrução de trajetórias, geometrias e modelos físicos. Para este trabalho, os valores calculados em simulação são comparados com as taxas medidas por dosímetros termoluminescentes (TLDs) em fantoma físico REMAB®. A partir da análise dos dados obtidos através da simulação e experimentação, dentre os 14 pontos estudados, observouse uma discrepância de até $8,2 \%$ dos valores de kerma encontrados. O fantoma geométrico implementado em código de Monte Carlo Geant4 foi devidamente validado e poderá ser usado, posteriormente, para a avaliação de doses em extremidades.
\end{abstract}

Keywords: Fantoma Geométrico; Geant4; Tomografia por Emissão de Pósitrons 


\section{INTRODUÇÃO}

Dados da Comissão Nacional de Energia Nuclear (CNEN) mostram existir no ano de 2014 cerca de 420 serviços de medicina nuclear, espalhados em todo o território nacional. São realizados mais de 50 procedimentos clínicos diferentes utilizando radiofármacos, com um total de 3 milhões de pacientes atendidos anualmente em todo o Brasil.

Segundo a Agência Internacional de Energia Atômica (AIEA), as últimas duas décadas, foram acompanhadas pela crescente demanda das modalidades de imagem funcional para diagnósticos, terapias, testes de acompanhamento de patologias e avaliação de recorrência de câncer (IAEA, 2008).

O elevado número de pacientes submetidos a exames em medicina nuclear e profissionais envolvidos nesta prática justifica esforços permanentes para a melhoria da qualidade dos diagnósticos e para a redução dos riscos radiológicos inerentes. Os profissionais em Medicina Nuclear que se encontram envolvidos em atividades ligadas a emissores de alta energia, são considerados como um grupo de maior exposição à radiação (BIXLER et Al. 1999).

Faz-se então necessária a análise da dosimetria destes trabalhadores, que são monitorados rotineiramente para avaliação de sua exposição externa à radiação - seja a partir do uso de anéis e pulseiras dosimétricas e/ou de filmes dosimétricos e dosímetros de bolso - já que a exposição de extremidades durante a administração de radiofármacos é considerada relativamente alta (COVENS et Al., 2010).

A simulação de Monte Carlo (MC) pode ser considerada uma excelente ferramenta de otimização desta prática, ao rever o uso correto da utilização de monitores pessoais e, a partir do momento em que obtém resultados com alta acurácia estatística em um curto espaço de tempo, podendo fazer variações de diferentes parâmetros que, em muitos casos, não podem ser facilmente mensuráveis em um experimento real.

O método de MC é utilizado como ferramenta auxiliar no desenvolvimento de novos experimentos, assim como na verificação de alguns resultados obtidos experimentalmente. Pode ser utilizado para simular o comportamento de sistemas físicos, matemáticos e também biológicos. Além disso, permite entender melhor os processos físicos nos quais se baseiam 
alguns aparatos experimentais, podendo estar ligado aos mais diversos segmentos da ciência e engenharia (como cromodinâmica quântica, fluxo de tráfego, reator nuclear e evolução estelar sistemas estes de geometria complexa). Em um experimento real, muitos parâmetros não podem ser mensuráveis ou não são facilmente testados, e o método de MC intervém como uma alternativa para testar o efeito de suas várias hipóteses antes da construção de um equipamento real (BONIFÁCIO, 2007; BORTOLINI, 2009; YORIYAZ, 2009).

As simulações computacionais desempenham um papel chave em Medicina nuclear, com aplicações que incluem o design de um equipamento, a reconstrução tomográfica de imagens, e a otimização de protocolos (SANTIN, 2003).

Neste trabalho, foi implementado no Geant4 - um programa para simulação em Monte Carlo um fantoma geométrico representando a mão do profissional envolvido no procedimento clínico, com dimensões de um antebraço de homem padrão, que permita uma posterior avaliação da exposição de extremidades.

O fantoma geométrico, apesar de ser uma representação menos realista que o fantoma de voxel, possui maior flexibilidade - sua geometria permite que uma simples definição de ângulos gerasse articulações em seus dedos, facilitando investigações em qualquer configuração que exija apenas a maleabilidade dos dedos.

Após a construção da geometria, foi feita a validação do fantoma geométrico através da obtenção de valores de kerma no ar com TLDs (dosímetros termoluminescentes), colocados em pontos preestabelecidos, em segmento de membro superior de um fantoma REMAB ${ }^{\circledR}$.

\section{MATERIAIS E MÉTODOS}

A primeira etapa do trabalho está relacionada à construção da geometria de simulação - o fantoma geométrico de mão e antebraço - a partir de sólidos geométricos simples.

A montagem do fantoma geométrico teve início após definição de quais estruturas seriam simuladas. Como não seria necessário considerar a articulação do pulso, os ossos do carpo não foram representados em simulação, sendo apenas considerados os demais ossos da mão (falanges e metacarpo) e antebraço (ulna e rádio). 
A simulação de Monte Carlo foi feita com o pacote Geant4, versão 9.6. Durante a modelagem no Geant4 foram utilizadas formas prismáticas e cilíndricas, por serem formas que melhor se aproximam das figuras de referência. Considerou-se então para a representação dos ossos, somente cilindros, assim como para os dedos; para projeção da região que compreende a palma da mão, foi usado um prisma retangular e para o segmento do antebraço foi utilizado um prisma de base elíptica.

Estas formas, ou modelos geométricos, encontram-se preestabelecidos em "bibliotecas" do Geant4. A representação geométrica no código é feita pela descrição de sólidos geométricos inseridos em um sistema de coordenadas cartesianas. Estes sólidos são posicionados espacialmente a partir de um volume inicial - também chamado volume-mãe (representando o tecido epitelial) -, onde deverão ser inscritas as demais estruturas que representam o tecido mole e o tecido ósseo (figura 1).

Figura 1: Fantoma geométrico com corte em uma das falanges para melhor observação das estruturas internas (tecido epitelial - branco; tecido mole-vermelho; tecido ósseo-amarelo).

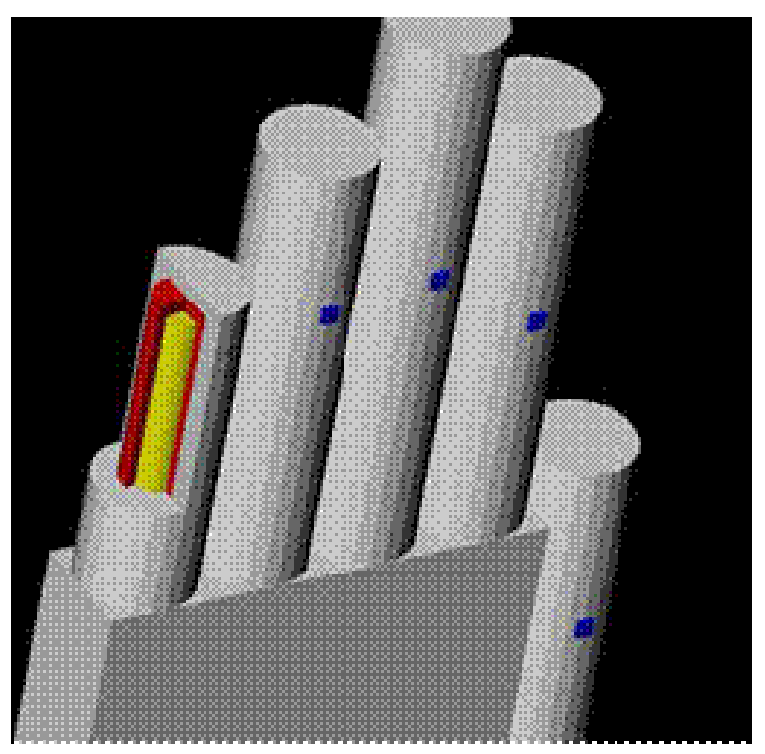

Para modelagem do fantoma durante a construção da geometria, foram utilizados materiais do ICRP 23 (tabela 1). As dimensões do fantoma estão dispostas na tabela 2. 
Tabela 1. Elementos que compõem os tecidos utilizados no fantoma (ICRP, 1975).

\begin{tabular}{cccc}
\hline \multirow{2}{*}{ Elementos } & \multicolumn{3}{c}{ Fração de massa (\%) } \\
\cline { 2 - 4 } & Ossos & Tecido Mole & Pele \\
\hline $\mathrm{H}$ & 0,07 & 0,10 & 0,1022 \\
$\mathrm{C}$ & 0,23 & 0,23 & 0,2693 \\
$\mathrm{~N}$ & 0,039 & 0,023 & 0,0426 \\
$\mathrm{O}$ & 0,49 & 0,63 & 0,5804 \\
$\mathrm{Na}$ & 0,0032 & 0,0013 & 0,00012 \\
$\mathrm{Mg}$ & 0,0011 & 0,00015 & $5,4 \mathrm{E}-05$ \\
$\mathrm{P}$ & 0,069 & 0,0024 & 0,0031 \\
$\mathrm{~S}$ & 0,0017 & 0,0022 & 0,0015 \\
$\mathrm{Cl}$ & 0,0014 & 0,0014 & 0,00253 \\
$\mathrm{~K}$ & 0,0015 & 0,0021 & 0,0008 \\
$\mathrm{Ca}$ & 0,099 & - & 0,0014 \\
$\mathrm{Fe}$ & $8,0 \mathrm{E}-05$ & $6,3 \mathrm{E}-05$ & $1,5 \mathrm{E}-05$ \\
$\mathrm{Zn}$ & $4,8 \mathrm{E}-05$ & $3,2 \mathrm{E}-05$ & $5,4 \mathrm{E}-06$ \\
$\mathrm{Rb}$ & - & $5,7 \mathrm{E}-06$ & $2,1 \mathrm{E}-09$ \\
$\mathrm{Sr}$ & $3,2 \mathrm{E}-05$ & $3,4 \mathrm{E}-07$ & $6,6 \mathrm{E}-07$ \\
$\mathrm{Zr}$ & - & $8,0 \mathrm{E}-06$ & $1,86 \mathrm{E}-06$ \\
$\mathrm{~Pb}$ & $1,1 \mathrm{E}-05$ & $1,6 \mathrm{E}-07$ & $2,8 \mathrm{E}-07$ \\
\hline Densidade $\left(\mathbf{g} / \mathbf{c m}^{\mathbf{3}}\right)$ & $\mathbf{1 , 4 8 6}$ & $\mathbf{0 , 9 8 7}$ & $\mathbf{1 , 1 0 5}$ \\
\hline
\end{tabular}

Tabela 2. Dimensões usadas na modelagem do fantoma de mão (FILHO, 2005).

\begin{tabular}{lc}
\hline Segmento & Tamanho $(\mathbf{c m})$ \\
\hline Comprimento do dorso da mão & 9,89 \\
Comprimento do médio & 10,43 \\
Largura da mão & 10,60 \\
Comprimento do polegar & 6,38 \\
Comprimento do antebraço & 30,0 \\
Espessura da mão & 3,0 \\
\hline
\end{tabular}


A especificação das peças anatômicas representando as falanges da mão foi formada por dois grupos de segmentos de sólidos cilíndricos. O primeiro segmento foi utilizado para representar as falanges proximais, e o segundo segmento as falanges média e distal.

Esta estrutura foi obedecida durante a construção dos segmentos que representaram os tecidos mole e epitelial, proporcionando aos dedos uma modelagem a partir de um conjunto de cilindros concêntricos, os quais retratavam suas estruturas internas (figura 2).

Esta configuração teve como exceção o polegar, onde um dos seguimentos cilíndricos representava as falanges (proximal, média e distal) e o segundo segmento representava o metacarpo. A partir da especificidade destes vértices é que o fantoma geométrico torna-se capaz de promover a simulação de movimentos, através da definição de ângulos nas articulações entre as falanges proximal e média.

Figura 2: Fantoma geométrico com articulações falângicas.

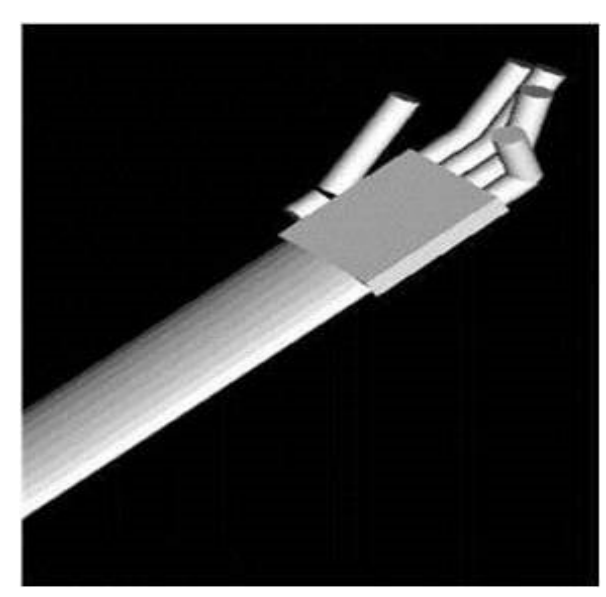

A característica de maleabilidade da mão pode ser considerada como de extrema relevância ao considerá-la como principal órgão eferente do homem. É esse o fato que possibilita a manipulação de objetos diferentes com eficácia precisão e força.

Como a construção da geometria de simulação foi voltada para modelagem da mão de trabalhador de Medicina Nuclear, em procedimento de injeção, apenas as articulações falângicas foram simuladas. 
O programa foi validado a partir da irradiação de um fantoma físico, com medições de kerma no ar com cristais termoluminescentes (TLDs) e comparação com valores obtidos na simulação.

Os critérios para validação do programa obedeceram aos parâmetros antropométricos de largura e comprimento, além de materiais, que compõem o fantoma REMAB ${ }^{\circledR}$ descritos pela ICRU 48 casca de acetato de celulose, contendo esqueleto humano, que permite o seu preenchimento com água (ICRU, 1992).

Apenas o segmento de mão e antebraço do fantoma REMAB ${ }^{\circledR}$ foi utilizado, já que se buscou implementar um fantoma para determinação da exposição de extremidades dos membros superiores (figura 3).

Figura 3: Segmento do Fantoma REMAB ${ }^{\circledR}$ utilizado.

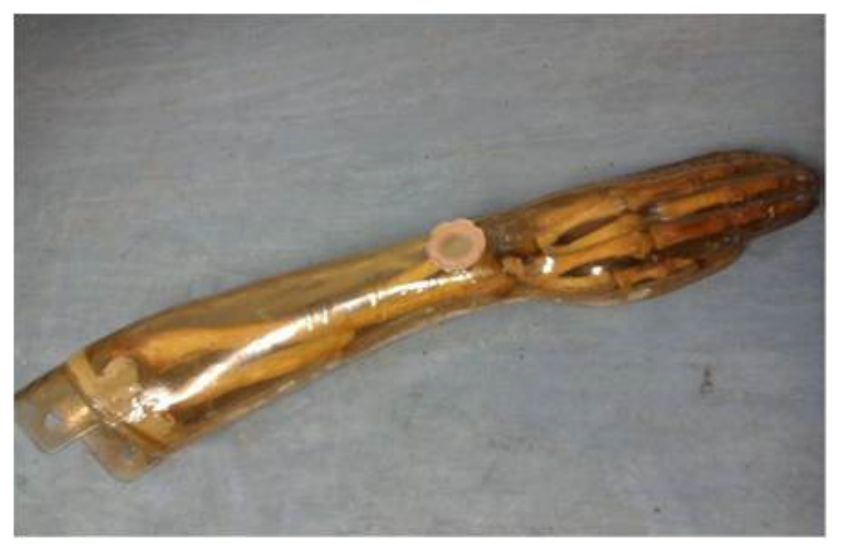

As dimensões do fantoma $\mathrm{REMAB}^{\circledR}$ foram obtidas com o auxílio de um paquímetro, para ajustar os valores da modelagem computacional e permitir a validação do programa. A partir de algumas poucas alterações em seu código, o fantoma geométrico permite o ajuste de suas dimensões e/ou materiais e elementos de acordo com as necessidades do usuário.

Para este trabalho, o fantoma simulado deveria estar imerso em ambiente composto por ar, além de apresentar suas articulações com os mesmos ângulos do fantoma REMAB ${ }^{\circledR}$.

Foi utilizado para dosimetria do fantoma de mão e validação da simulação um total de 90 TLDs, retirados de um lote homogêneo (desvio padrão das respostas inferior a 3\%) de TLD-100 (LiF:Mg, Ti), de dimensões 0,9 $\mathrm{mm}$ x 0,9 $\mathrm{mm}$ x 3,0 mm, pertencentes ao Laboratório de Dosimetria Termoluminescente (LDT) do Instituto de Radioproteção e Dosimetria (IRD). 
Destes, 10 TLDs foram irradiados no ar, em condições de equilíbrio eletrônico, em um campo padronizado de uma fonte de ${ }^{137}$ Cs da Divisão de Dosimetria (DIDOS/IRD), para que fosse obtido o fator de calibração para determinação dos valores de kerma no ar.

Os TLDs restantes foram separados em diferentes grupos, um deles contendo 10 TLDs para medição do background, e os outros grupos - contendo 5 TLDs cada - colocados em invólucros plásticos, para que fossem posicionados sobre o fantoma (figura 4) irradiado pela mesma fonte utilizada para obtenção do fator de calibração para kerma no ar.

Figura 4: TLDs sendo posicionados sobre o fantoma, indicados pelas setas.

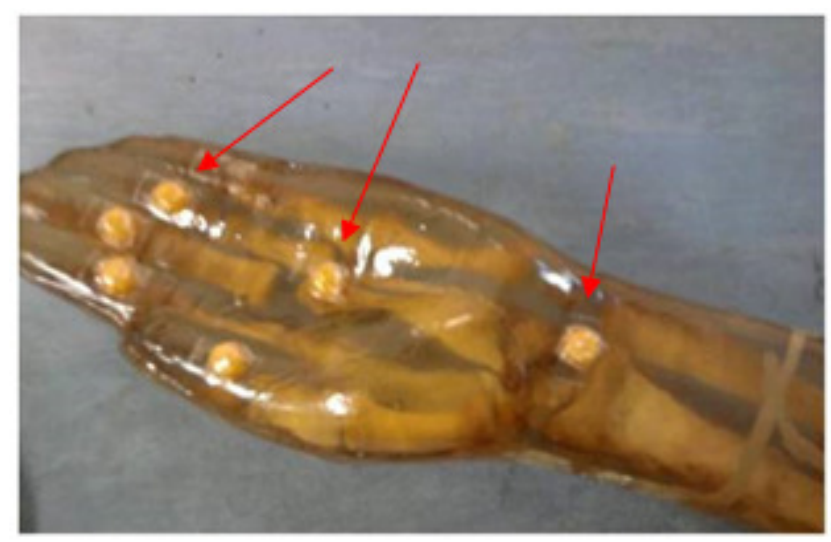

Cada grupo de TLDs e cada TLD foram identificados numericamente para que fossem reconhecidos após sua leitura e avaliação. Os grupos, numerados de 1 a 14, foram colocados no fantoma de acordo com o posicionamento indicado na figura 5.

Figura 5: Posições escolhidas para colocação dos TLDs e posterior obtenção dos valores de kerma. (A) TLDs anteriores; (B) TLDs posteriores. 


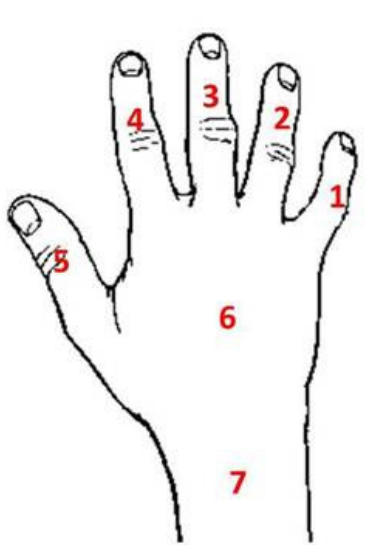

(A)

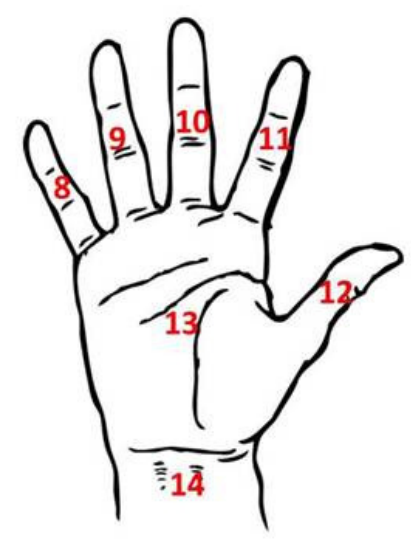

(B)

A preparação, leitura e avaliação dos dosímetros utilizados seguiram os procedimentos rotineiros de laboratório. O mesmo padrão de posicionamento dos TLDs no fantoma REMAB® foi utilizado no fantoma geométrico.

Para validação do programa, o fantoma teve a configuração de ângulo das articulações falângicas modelada de forma que obedecesse a geometria de posicionamento de dedos do fantoma físico mão com dedos esticados (figura 6).

Figura 6: Fantoma com dedos esticados e volumes TLDs, obedecendo à mesma configuração que o fantoma físico REMAB囚.

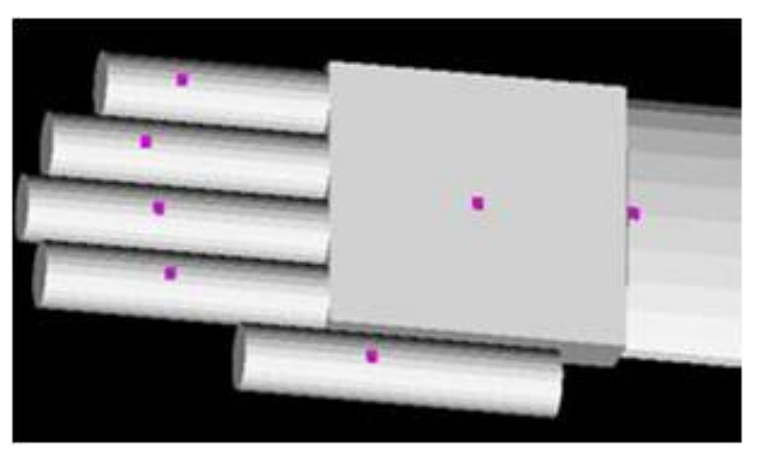

Os TLDs gerados em simulação possuem as mesmas dimensões de um TLD real utilizado. Os dados obtidos em laboratório foram comparados com os valores de kerma no ar obtidos pela simulação do fantoma REMAB $^{\circledR}$ em código Geant4, obedecendo a critérios como posicionamento em relação à fonte de ${ }^{137} \mathrm{Cs}$ e a energia do feixe (figuras 7 e 8). 
Figura 7: Fantoma posicionado para irradiação com feixe de ${ }^{137}$ Cs.

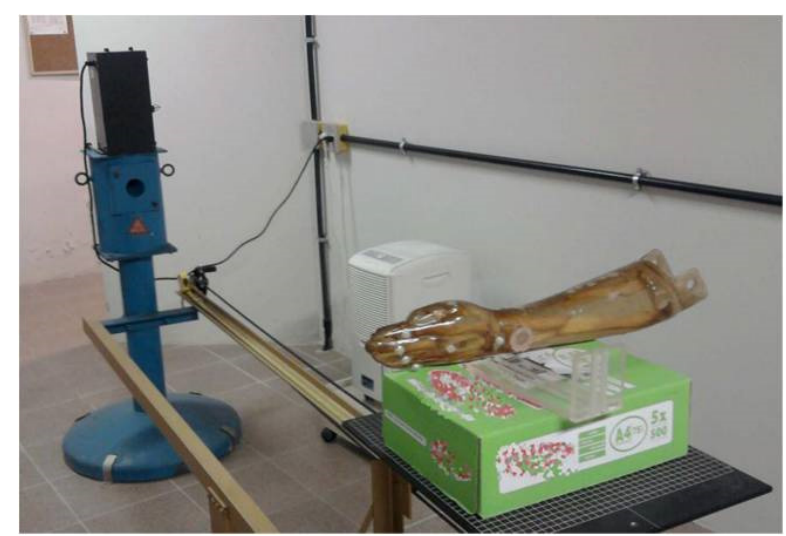

Figura 8: Arranjo com placa de acrílico (PMMA) inserida à frente do fantoma para garantia do equilíbrio eletrônico.

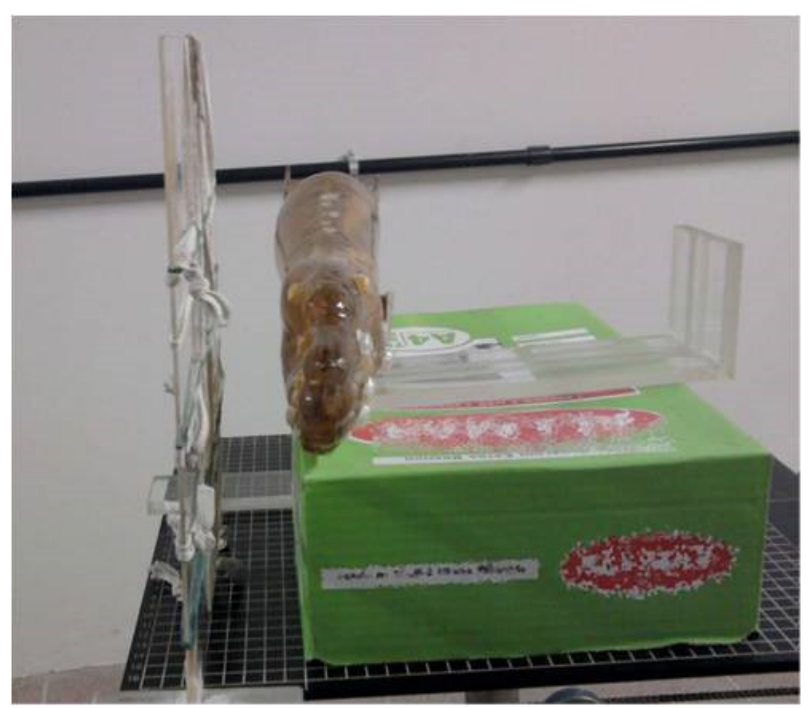

A fonte de ${ }^{137}$ Cs foi simulada como uma fonte plana, de modo que, a dimensão do feixe de partículas gerado fosse suficiente pra cobrir a área do fantoma geométrico onde os TLDs estavam posicionados (figura 9).

Figura 9: Simulação do Fantoma REMAB ${ }^{\circledR}$. Visualização do campo de irradiação. 


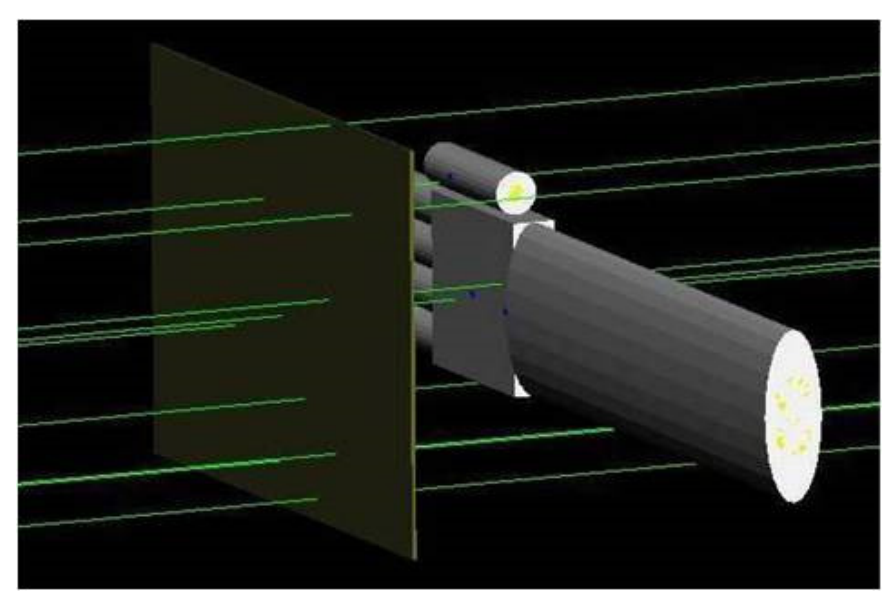

A avaliação das incertezas na parte experimental foi feita por meio do desvio padrão das cinco leituras dos TLDs posicionados em cada um dos 14 pontos de monitoração. Na simulação as incertezas foram obtidas por meio do desvio padrão dos valores de kerma obtidos para diferentes sementes de números aleatórios gerados.

\section{RESULTADOS E DISCUSSÕES}

Os valores de kerma no ar obtidos em código Geant4, com o uso de uma fonte plana de ${ }^{137} \mathrm{Cs}$, são observados na Tabela 3.

Tabela 3. Valores de kerma no ar por fóton, obtidos a partir da simulação de fonte de

\begin{tabular}{|c|c|c|c|c|}
\hline & Volume & TLD & $\begin{array}{c}\text { kerma } \\
\text { (nGy/fóton) }\end{array}$ & $\begin{array}{c}\text { Desvio Padrão } \\
\text { (nGy/fóton) }\end{array}$ \\
\hline \multirow{7}{*}{ 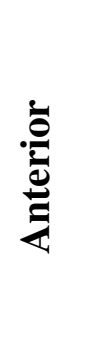 } & Mínimo & 1 & 7,83E-06 & $2,26 \mathrm{E}-09$ \\
\hline & Anelar & 2 & $7,85 \mathrm{E}-06$ & $2,25 \mathrm{E}-09$ \\
\hline & Médio & 3 & 7,84E-06 & $2,25 \mathrm{E}-09$ \\
\hline & Indicador & 4 & 7,85E-06 & $2,25 \mathrm{E}-09$ \\
\hline & Polegar & 5 & $7,85 \mathrm{E}-06$ & $2,45 \mathrm{E}-09$ \\
\hline & Palma da mão & 6 & 7,91E-06 & 2,21E-09 \\
\hline & Pulso & 7 & 7,88E-06 & 2,21E-09 \\
\hline \multirow{2}{*}{ 皇 } & Mínimo & 8 & $6,89 \mathrm{E}-06$ & $2,41 \mathrm{E}-09$ \\
\hline & Anelar & 9 & $6,95 \mathrm{E}-06$ & 2,39E-09 \\
\hline
\end{tabular}




\begin{tabular}{cccc} 
Médio & 10 & $6,92 \mathrm{E}-06$ & $2,39 \mathrm{E}-09$ \\
Indicador & 11 & $6,91 \mathrm{E}-06$ & $2,40 \mathrm{E}-09$ \\
Polegar & 12 & $6,95 \mathrm{E}-06$ & $2,39 \mathrm{E}-09$ \\
Dorso & 13 & $7,29 \mathrm{E}-06$ & $2,31 \mathrm{E}-09$ \\
Pulso & 14 & $6,88 \mathrm{E}-06$ & $2,37 \mathrm{E}-09$ \\
\hline
\end{tabular}

Para a obtenção dos valores de kerma no ar nos pontos de análise, se faz necessário conhecer a quantidade de fótons incidentes na superfície dos volumes dos TLDs - gerados durante o decaimento da fonte de ${ }^{137} \mathrm{Cs}$ - para um intervalo de tempo previamente estabelecido.

Os valores de kerma no ar e seus respectivos desvios padrão irradiados em ${ }^{137} \mathrm{Cs}$ para a validação do programa podem então ser encontrados na Tabela 4.

Tabela 4. Comparação entre os valores de kerma no ar obtidos a partir da simulação e experimentação para fonte de ${ }^{137} \mathrm{Cs}$.

\begin{tabular}{|c|c|c|c|c|c|c|c|}
\hline \multirow{2}{*}{\multicolumn{2}{|c|}{ Volume }} & \multirow[b]{2}{*}{ TLD } & \multicolumn{2}{|c|}{ Simulação } & \multicolumn{2}{|c|}{ Experimental } & \multirow{2}{*}{$\begin{array}{c}\text { Desvio entre } \\
\text { valores } \\
\text { experimental e } \\
\text { simulação }(\%)\end{array}$} \\
\hline & & & $\begin{array}{r}\text { Kerma } \\
(\mathrm{mGy})\end{array}$ & $\begin{array}{c}\text { Desvio } \\
\text { Padrão } \\
(\mathbf{m G y )}\end{array}$ & $\begin{array}{c}\text { Kerma } \\
(\mathrm{mGy})\end{array}$ & $\begin{array}{c}\text { Desvio } \\
\text { Padrão } \\
\text { (mGy) }\end{array}$ & \\
\hline \multirow{7}{*}{ 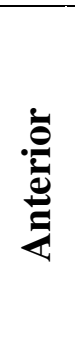 } & Mínimo & 1 & 2,00 & 0,00058 & 1,88 & 0,11 & 6,6 \\
\hline & Anelar & 2 & 2,01 & 0,00058 & 1,89 & 0,08 & 6,4 \\
\hline & Médio & 3 & 2,01 & 0,00058 & 1,90 & 0,08 & 5,6 \\
\hline & Indicador & 4 & 2,01 & 0,00058 & 1,95 & 0,01 & 3,1 \\
\hline & Polegar & 5 & 2,01 & 0,00063 & 1,88 & 0,09 & 7,0 \\
\hline & Palma & 6 & 2,03 & 0,00057 & 2,00 & 0,15 & 1,2 \\
\hline & Pulso & 7 & 2,02 & 0,00056 & 2,03 & 0,16 & 0,6 \\
\hline \multirow{7}{*}{ 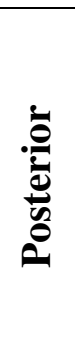 } & Mínimo & 8 & 1,76 & 0,00062 & 1,63 & 0,14 & 8,2 \\
\hline & Anelar & 9 & 1,78 & 0,00061 & 1,71 & 0,10 & 4,2 \\
\hline & Médio & 10 & 1,77 & 0,00061 & 1,71 & 0,07 & 3,6 \\
\hline & Indicador & 11 & 1,77 & 0,00061 & 1,66 & 0,16 & 6,6 \\
\hline & Polegar & 12 & 1,78 & 0,00061 & 1,87 & 0,12 & 4,9 \\
\hline & Dorso & 13 & 1,87 & 0,00059 & 1,58 & 0,05 & 18,1 \\
\hline & Pulso & 14 & 1,76 & 0,00061 & 1,44 & 0,10 & 22,2 \\
\hline
\end{tabular}

A figura 10 mostra uma comparação entre os valores obtidos experimentalmente e através de simulação em código Geant4 já observados na tabela 4. 
Figura 10: Dados experimentais e de simulação referentes à validação do programa.

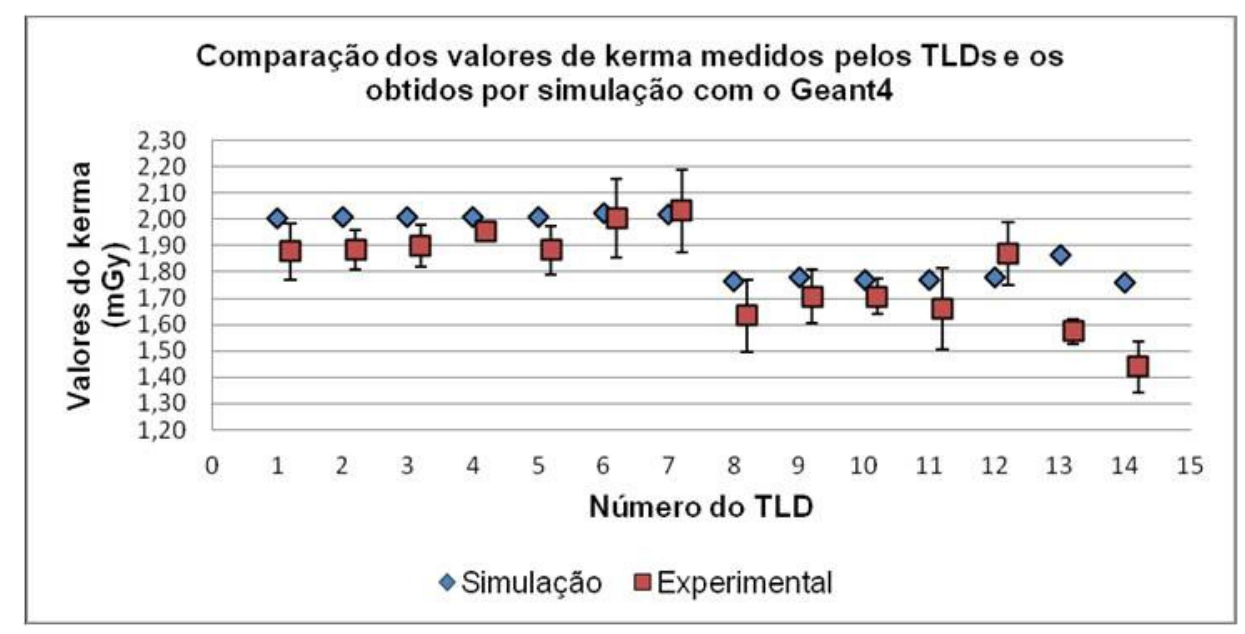

A incerteza máxima para os valores estabelecidos nas medidas experimentais foi de aproximadamente $10 \%$. Esta incerteza foi obtida através da análise de cinco dosímetros para cada ponto estabelecido na mão (figura 3.10). Nos resultados da simulação, foram consideradas apenas as incertezas do tipo A. Contribuições de alguns parâmetros, tais como a distinção entre os volumes reais e volumes utilizados no fantoma durante a simulação não foram considerados, bem como as incertezas associadas às características dos materiais que o compõem.

Os dados observados na tabela 4 mostram uma discrepância entre os valores de até 8,2\%, com exceção dos dois últimos pontos (volumes dorso e pulso posterior) onde as discrepâncias atingiram valores de 18 e $22 \%$, respectivamente.

Uma das razões para os valores de kerma no ar nos segmentos dorso e pulso posterior apresentarem diferenças significativas entre os valores experimentais e simulados, deve-se ao fato do fantoma físico possuir em sua estrutura todos os segmentos que compõem o tecido esquelético do membrosuperior, contando assim com os ossos que fazem parte do carpo (região do pulso).

Durante a modelagem do fantoma geométrico, os segmentos representativos dos ossos que compõem a região do pulso não foram simulados, assim como os ângulos promovidos pelo posicionamento dos ossos de metacarpo, já que para o objetivo do trabalho seria necessário somente a representação das articulações falângicas para movimentação dos dedos e obtenção de uma geometria que permita a reprodução da administração de radiofármacos através de seringa 
de injeção. A existência dos ossos do carpo e dos ângulos que os ossos do metacarpo formam entre si, observados no fantoma físico, funcionariam como uma blindagem à passagem de radiação em alguns pontos da região de dorso e pulso.

A partir dos dados da tabela 4 e figura 10, podemos concluir que a implementação do fantoma geométrico em código Monte Carlo Geant4 foi validada, já que os valores para kerma encontrados em simulação e experimentação mostram-se compatíveis. Observamos também que os valores de kerma obtidos pelos TLDs colocados na parte anterior do fantoma foram ligeiramente maiores que os valores de kerma encontrados nos TLD situados na parte posterior. Isso se deve ao fato do próprio fantoma contribuir para a atenuação à passagem da radiação de uma região a outra.

\section{CONCLUSÕES}

O fantoma geométrico implementado em código de Monte Carlo Geant4 foi devidamente validado, sendo possível a obtenção de resultados para doses em extremidades durante administração de radiofármacos utilizando seringas de injeção.

Contudo, a discrepância entre os dados obtidos para kerma no ar em fantoma geométrico e fantoma $\mathrm{REMAB}^{\circledR}$ utilizado em laboratório durante a validação apresentou valores de até $20 \%$ em alguns pontos. Esta diferença está associada à desigualdade das geometrias dos fantomas utilizados - simulado e experimental - nos pontos considerados.

Com relação à exposição à radiação, o conhecimento sobre as estimativas de dose mostra-se de extrema relevância quanto às práticas de radioproteção dos profissionais inseridos nos serviços de Medicina Nuclear. Em um próximo trabalho este fantoma será utilizado para a avaliação de doses de extremidades de trabalhadores ocupacionalmente expostos que realizam práticas de Tomografia por Emissão de Pósitrons (PET).

\section{REFERÊNCIAS}


BIXLER, A.; SPRINGER, G.; LOVAS, R. Practical Aspects of Radiation Safety for Using Fluorine-18. Journal of Nuclear Medicine Technology. Vol. 27. No. 1. pp 14-16, 1999.

BONIFÁCIO, D. A. B., Validação do Geant4 para produção e detecção de raios X na faixa de energia de radiodiagnóstico. Dissertação de Mestrado, Universidade de São Paulo. São Paulo, 2007.

BORTOLINI, A. Avaliação de um Sistema de Colimação do Protótipo de um Tomógrafo Computadorizado por Feixe de Prótons por Simulações de Monte Carlo. Dissertação de Mestrado, Pontifícia Universidade Católica do Paraná. Curitiba, 2009.

COVENS, P.; BERUS, D.; VANHAVERE, F.; CAVELIERS, V., The Introduction of automated dispensing and injection during PET procedures: A step in the optimization of extremity doses and whole-body doses of nuclear medicine staff. Radiation Protection Dosimetry. Vol. 140, No. 3, pp. 250-258, 2010.

CNEN - Comissão Nacional de Energia Nuclear. Instalações autorizadas - Medicina Nuclear, Disponível em: <http://www.cnen.gov.br/seguranca/cons-ent-prof/lst-entidades-autcert.asp?p_ent=42\&d=Medicina\%20Nuclear $>$, acessado em 25 de agosto de 2014 .

IAEA - International Atomic Energy Agency. Radiation Protection In Newer Medical Imaging Techniques: PET/CT. Safety Reports Series, No. 58. IAEA, Vienna, 2008.

ICRP - International Comission on Radiological Protection. Report on the Task Group on Reference Man. ICRP Publication 23, Oxford: Pergamon Press; Ann. ICRP, 1975. FILHO, H. V. Avaliação da Força de Preensão Palmar Plena e Pulpo-lateral: relação com a antropometria da mão e posições de trabalho. Dissertação de Mestrado. Universidade Estadual Paulista, Bauru - SP, 2005. 
ICRU - International Comission on Radiation Units and Measurements. Phantoms and Computational Models in Therapy, Diagnosis and Protection. ICRU-48, Bethesda, MD, 1992.

SANTIN, G.; STRUL, D.; LAZARO, D.; SIMON, L.; KRIEGUER, M.; VIEIRA MARTINS, M.; BRETON, V.; MOREL, C., GATE: A Geant4-Based Simulation Platform for PET and SPECT Integrating Movement and Time Management. IEEE Transactions on nuclear science. Vol. 50, No. 5. pp.1325-1329, 2003.

YORIYAZ, H. Método de Monte Carlo: princípios e aplicações em Física Médica. Revista Brasileira de Física Médica. Vol. 3, No.1, pp.141-149, 2009. 